\title{
Reflections on the past two decades of neuroscience
}

\author{
Danielle S. Bassett \\ Kathleen E. Cullen \\ Simon B. Eickhoff \\ Martha J. Farah \\ Yukiko Goda \\ Patrick Haggard \\ Hailan Hu \\ Yasmin L. Hurd \\ Sheena A. Josselyn \\ Baljit S. Khakh \\ Jürgen A. Knoblich \\ Panayiota Poirazi \\ Russell A. Poldrack \\ Marco Prinz \\ Pieter R. Roelfsema \\ Tara L. Spires-Jones \\ Mriganka Sur \\ Hiroki R. Ueda
}

The first issue of Nature Reviews Neuroscience was published 20 years ago, in 2000. To mark this anniversary, in this Viewpoint article we asked a selection of researchers from across the field who have authored pieces published in the journal in recent years for their thoughts on notable and interesting developments in neuroscience, and particularly in their areas of the field, over the past two decades. They also provide some thoughts on current lines of research and questions that excite them.

Danielle S. Bassett. Science is a culture as much as it is a practice in the acquisition and curation of knowledge. As time passes, the field changes its culture and practice. As a culture, neuroscience is now composed of a more diverse group of scientists, along the dimensions of sex, gender, sexual orientation, race, ethnicity and disability. With the creation of AnnesList (anneslist.net), Women in Neuroscience Repository (winrepo.org) and BiasWatchNeuro (biaswatchneuro.com), there exist resources to increase awareness of the work of women scientists, and to encourage a greater balance in the speaker line-ups of conferences and symposia. Yet, explicit bias exists now as it did in 2006 when transgender Professor Ben A. Barres (Stanford University) wrote his commentary for Nature entitled 'Does Gender Matter?' And implicit bias is perhaps even more pervasive, as reflected in peer review, paper acceptance rates, grant funding and a growing (rather than diminishing) undercitation of women in the reference lists of neuroscience journal articles in the past 20 years. Each of us as citizens of science can choose to own and mould our culture to realize a more equitable future.

As a practice in the acquisition and curation of knowledge, neuroscience has expanded upon prior paradigms. In human neuroscience, I have been particularly struck by a concerted 
effort to move beyond the activation studies that were characteristic of the brain-mapping era. Leading from that point in scholarly space, two particularly pioneering paths of formal study extend: that of neural representations and that of network systems. The former considers the pattern of activity across neural units that encodes an object, concept or state of information. The latter considers the pattern of connections between neural units that can support the transmission of information and the modulation of network state in a manner that alters the repertoire of computations accessible to a given neural population. The twin paths of representation and transmission move beyond the mapping of a country's borders, to record a city's statistics and the transportation networks along which traffic flows.

To me, the question that creates giant resonances when dropped into the liquid medium of conceptual space is: how do we combine these twin paths to understand the transmission of representations in the network of the brain? Although answering that question will surely build on the prior paradigms, neither seems equipped to provide a complete solution; the former offers the dots within, whereas the latter offers the lines between. Yet obtaining such a solution is important for deciphering the mechanism of the brain's fundamental goal: information processing. The efforts toward such a solution that I find particularly promising are those that move beyond descriptive characterization or correlative approaches, and towards theory; that is, theory instantiated in an interpretable and generalizable mathematical model that encodes a conceptual principle, and theory as supported by (but not composed of) computational and machine learning approaches applied to data shared publicly in the spirit of open science. Progress along this tack will require more interdisciplinary graduate training programs that provide equal depth in theory and experiment.

Kathleen E. Cullen. Traditionally, the field of neuroscience has asked the question: how is sensory input (afference) transformed into motor output (efference) to generate appropriate behaviour? This conceptual framework - initially made popular by Sherrington - has long served as a common foundation for systems neuroscientists. A fundamental development over the past two decades, however, has been a collective realization that it is necessary to reverse this traditional perspective. A series of recent studies has established that motor signals profoundly influence sensory processing during voluntary behaviour. In particular, experiments across sensory systems have provided circuit-level insight into how motorrelated inputs to sensory areas selectively cancel self-generated sensory inputs. This neural strategy underlies an essential property of the brain, namely its ability to distinguish externally applied (exafferent) from self-generated (reafferent) sensory inputs. For instance, work by my group has established that neurons at the first central stage of vestibular processing preferentially encode sensory exafference. Moreover, researchers studying other sensory pathways (for example, visual, somatosensory, and auditory) have shown suppression of reafferent sensory information by motor-related inputs at higher levels of sensory processing, including in the cortex.

Indeed, much of the sensory input that we experience in our everyday lives is reafferent since it is generated by our own behaviour. The brain's ability to predict the sensory consequences of our actions based on motor-related inputs is vital for both perceptual stability and accurate motor control. This computational strategy also provides the flexibility required for fine-tuning and updating relationships between motor signals and resultant sensory feedback in order to compensate for changes to our body or the environment. Furthermore, the ability to distinguish between sensory exafference and reafference is a 
hallmark of higher-level perceptual and cognitive processing that is central to our subjective awareness of initiating and controlling our own volitional actions in the world (that is, our sense of agency). In this context, a fundamental question logically arises: how exactly does the brain actually predict the sensory consequences of our actions in a dynamically changing world?

Two particularly exciting lines of research are currently addressing this question. First, emerging technologies including high-density recording across brain regions and the ability to target perturbations in neural circuits are providing new insight into how specific brain regions, such as the cerebellum, actually perform this essential computation at the level of single neurons. Second, recent advances in our understanding of sensory processing during natural behaviours have motivated the investigation of neural circuits in real-world environments. For example, the brain must recalibrate its encoding of sensory information in conditions in which relationships between motor signals and reafferent sensory feedback are altered. Yet, studies of the neural basis of our sense of direction and place have traditionally used virtual reality set-ups that unintentionally generate persistent sensorimotor mismatches compared to natural navigation and/or recorded only coarse measurements of behaviour (for example, head position). On-going experiments focused on understanding circuit-level neural coding strategies during navigation - using more precise and comprehensive measurements the complex animal behaviour - are now beginning to elucidate the neural computations underling navigation in changing real-world environments.

Simon B. Eickhoff. Cognitive and systems neuroscience has gone through a period of explosive growth over the past 2-3 decades following the advent, expansion and maturation of in vivo neuroimaging. Among the many noteworthy developments over that period, the increase in sample size certainly stands out. In the late 1990s, MRI studies involving fewer than 10 individuals were the norm. However, this number has grown continuously and nowadays studies with several dozen if not hundreds of participants are common. Moreover, in particular for task-free data, analyses of 10,000s of individuals have become feasible due to increased data-sharing and large-scale efforts such as the UK Biobank. Although these developments could be rightfully attributed to increasing awareness about power and replicability, another main driver is the evolution of study objectives. Earlier studies often focused on robust effects that are often well discernable even in individuals, but paradigms became more complex and differences between conditions became increasingly subtle, highlighting the aforementioned concerns. Moreover, although the search for the 'neural correlates of...' in group analyses has long dominated the field, more recently, inter-individual differences have come more into focus.

In this context, one of the effects, and further driver, of large sample sizes is the growing focus on individual inference using machine-learning. The mass-univariate investigation of within-sample associations is being increasingly abandoned in favour of multivariate models that are better suited for capturing the richness of MRI data. The latter also allow the prediction of inter-individual differences in phenotypical (for example, behavioural) characteristics in new, previously unseen individuals. Machine-learning approaches may thus hold the key towards delivering on some of the promises that have been abundant in the literature ever since the start of neuroimaging. Throughout the decades, many neuroimaging studies were ultimately motivated by clinical issues, which can hardly be realized by group-mean differences but may become possible using multivariate, predictive 
approaches. Even though further maturation and consolidation of the field is needed, it has brought real-life applications of neuroimaging research into reach.

Finally, although hardly a new development but rather a controversy going back 150 years to Broca and Lichtheim, the continued change in focus between localizing and connectionist perspectives is intriguing. The former became massively popular with the ability of task-based functional MRI (fMRI) to discern differences in activity between conditions, whereas the latter surged with the development of methods for studying structural and functional connectivity in vivo. More recently, novel methods for in vivo brain cartography at the level of groups or individuals on the one hand, and increasing focus on network-theory and dynamical system modelling on the other, have further advanced both perspectives. What is missing, though, and hence remains a key challenge for the future, are unified accounts for regional segregation and system-wide interactions in the brain. How to define the building blocks of the human brain and determine their likely context-dependent integration into larger systems, their inter-individual variability and their relationship to behaviour continue to be grand but crucial challenges.

Martha J. Farah. For most of the long history of neuroscience, the field has been defined by two main goals: to understand the brain and to alleviate neurological and psychiatric disorders. These two goals have recently been joined by a third: to enhance diverse nonmedical endeavours. New subjects of study with the prefix 'neuro' have proliferated, from neuroaesthetics to neurowarfare. This historical turn in the uses of neuroscience arrived right around the turn of the new century, so we are now about 20 years into the era of 'neuroeverything'.

The idea of neuroeverything understandably evokes eye rolls, within neuroscience and without. Many of these exuberant interdisciplinary connections will not turn out to be useful, but I believe that some will. Why? Because neuroscience is - in principle - relevant to any field that seeks to understand, predict or influence human behaviour. Whether and when it becomes relevant in practice is an empirical issue, but I see no reason to rule it out yet. Indeed, there are glimmerings of real progress in some of these fields.

Neurolaw took, as its initial focus, questions of criminal responsibility and punishment. Neuroscience has helped shore up the rationale for separate standards of punishment for juvenile offenders, given their incomplete brain development, and has been cited in US Supreme Court opinions on the matter. Neurolaw now encompasses issues beyond responsibility, reflecting the law's many intersections with psychology and behaviour. For example, cases involving claims of pain are common in the US legal system, with hundreds of billions of dollars in awards at stake each year. Pain is, of course, invisible, and there are obvious motivations for malingering. Brain imaging research on pain has revealed potential biomarkers, which may eventually prove useful in such cases (indeed they have already been used, but this was undoubtedly premature). Neuroeducation has evolved beyond vague, inprinciple statements about brains and learning to specific new insights about how children learn to read and do maths, and has delineated different types of learning difficulties and ways to individualize instruction based on these types. Neuromarketing is based on the idea that people often cannot or will not articulate their true preferences, whether for product packaging or political speeches, but that neural correlates of emotion and attention offer a new window on their inclinations. The academic literature backs up many of these claims. Companies around the world use neuromarketing, and political campaigns in a number of counties are reported to have consulted neuromarketers. 
Has neuroscience revolutionized the way we teach, do business or pursue justice? of course not, but that is an unreasonably high bar. Has it contributed to progress in these previously unrelated fields? My answer is a cautious, provisional "yes". The long-term impact of neuroscience on these and other fields remains to be seen, but what else would we expect after scarcely 20 years? The history of neurology and psychiatry shows that applying neuroscience to complex problems is slow and hard. Patience and an open mind are necessary, if not sufficient, for progress. So, for the time being, let us resist rolling our eyes.

Yukiko Goda. Neuroscience research over the past two decades has been catapulted by technological advances enabling manipulation and monitoring of neural activity in behaving mice at ever-higher spatial and temporal resolution over increasingly broad brain areas. Such developments have dramatically stimulated circuit-based analysis of the neural systems underlying cognitive functions and behaviour. Omics approaches transcriptomcis, proteomics and connectomics - are also providing novel views into the molecular and cellular architecture of the brain, revealing an extraordinary degree of complexity of individual cell types and their subcellular compartments across different brain areas and brain states.

In synapse biology, the past two decades have witnessed substantial insights into the molecular underpinnings of synapse organization and plasticity. We have learned, for example, how synapse specificity is driven by a combinatorial synapse adhesion code, that a neuron can pack different transmitters into single synaptic vesicles, sometimes even both glutamate and GABA, and that the principal mediators of synaptic transmission can dynamically move beyond the boundaries of individual synapses. Local protein translation, whose occurrence in dendrites is now well recognized, may also occur in presynaptic terminals. Collectively, the findings underscore the broad plasticity repertoire possessed by synapses. Dendritic spine plasticity during learning has continued to attract attention over the past two decades. The causal relationship between learning and spine structural changes has helped establish the hypothesis originally proposed by Donald Hebb that synapses are the building blocks of memory.

Excitatory principal neurons typically carry tens of thousands of synapses, each capable of expressing various forms of plasticity. A key issue is to close the widening gap between our circuit-level understanding of various neural systems, which is gaining momentum, and our understanding of the behaviour of individual synapses constituting the network. For example, how might synapse-specific Hebbian plasticity drive memory engrams that are represented by cellular networks? Furthermore, given our increasing understanding of Hebbian plasticity mechanisms, how do neighbouring synapses interact and balance distinct forms of synaptic plasticity, in some cases involving communication to the nucleus, to impact dendritic computation? Also, in contrast to spine plasticity, little is known of the contribution of presynaptic plasticity to particular circuit functions and related behaviours.

Further cell biological insights into the workings of synapses are also warranted. For example, super-resolution microscopy has provided a glimpse into the adhesive organization of a synapse where presynaptic release sites are aligned to nanoclusters of postsynaptic scaffolds. How plastic are such nano-columns and how are they regulated to guide transmitter release? Moreover, besides the presynaptic and the postsynaptic mechanisms, a better understanding of the contribution of glial signalling and the extracellular matrix that occupy the interstitial space would be crucial, as are the roles of 
neuropeptidergic signalling. Finally, the vast number of synapses in the brain are highly heterogeneous, even in the same neuron. Deciphering how synapse diversity arises in the context of specific circuit functions might shed new insights into synaptic information processing in the brain and may help in tackling diseases linked to synapse dysfunction, of which there are many and with mechanisms yet to be clarified.

Patrick Haggard. Since classical times, scientists have noted how human actions on the one hand seem to have a degree of unpredictability, and on the other hand are accompanied, at least sometimes, by a subjective feeling of being open, or 'up to me'. These observations often lead to theories of human action being somehow exceptional, or escaping from normal mechanistic causation. Advances in theory and methods over the past 20 years have shown that several key aspects of human voluntariness can be explained neuroscientifically, pushing back against exceptionalist theories.

Important progress has come from studies on decision-making. The introduction of formal decision theory into perceptual neuroscience, notably through drift diffusion models, turned out to be surprisingly informative in understanding internally generated, as well as stimulus-guided actions. For example, when stimulus evidence is ambiguous, internal motor noise can still drive a decision variable over the action threshold. A causal role of biological noise in voluntary action remains difficult to reconcile with our subjective feelings of deciding and controlling. However, being clear about the role of noise can at least help to distinguish the putative signals - internal drives, goals and dispositions - that underlie voluntary action.

A second area of progress comes from reinforcement learning ( $R L)$. Action choices are often based on learning from experience what worked previously in similar contexts. The past two decades have seen increasing focus on RL in human action. 'Model-based' RL can be viewed as an explicit account of how thinking translates into acting - perhaps the fundamental challenge of voluntary action. Exploratory or exploitative choices in actionoutcome learning may correspond to the innovative or creative aspect of human voluntary action, and to more routine and habitual aspects, respectively. The significance of RL lies in emphasising that humans learn to be voluntary. The movements of newborn infants seem purposeless, yet healthy adults learn to control highly complex movements, so as achieve desired outcomes. Interestingly, childhood amnesia means that we generally cannot remember the period of our lives where we lacked voluntary agency. Recent learning studies suggest that experienced linkage between action and outcome is boosted immediately after an error, suggesting an important connection between sense of agency and persistence in trying.

Voluntary action lies at the nexus of the stimulus-response chain and the actionoutcome chain. The past two decades have seen substantial progress in both areas, often driven by the combination of computational models, and mechanistic studies with animals. The space that volition must occupy is therefore more clearly defined. However, we still know little about how people select and maintain their goals. For this reason, recent neuroscientific developments have not undermined concepts of individual psychological autonomy, but rather described how such autonomy may arise, how it is acquired, and how it is deployed. The tension between what neuroscience teaches us about our volition, and what we are taught by cultural traditions and by our own subjective experience, remains to be resolved. 
Hailan Hu. One area I have seen booming is psychiatric neuroscience, the research into psychiatric disorders. Unlike neurological disorders, which are often associated with discrete anatomical lesions, psychiatric disorders used to be thought of as diseases of unknown aetiology. Indebted to many new mechanistic discoveries, the paradigm is shifting and the boundary between neurology and psychiatry is becoming less distinct. This new paradigm is led by revolutionary tools for brain imaging and manipulations, which permit direct testing, with unprecedented temporal and spatial precision, of the causal relationship between the activity of specific neural circuits and behavioural or disease states. In particular, aided by optogenetics or chemeogenetics, researchers can now mimic or mitigate aspects of different psychiatric states, including obsessive-compulsive disorder, addiction and depression, in animal models, by activating or inhibiting relevant neural circuits. By targeting these neural circuits, researchers can rigorously tackle the aetiology of psychiatric disorders, characterizing neural activity patterns and pinpointing molecular and cellular abnormalities in relevant brain areas.

Another interesting and exciting development in recent decades is the rise of social neuroscience, which explores the neurobiological mechanisms of social behaviour. Since social behaviour involves dynamic interactions between more than one animal or human, there are inevitably more variables that affect behavioural outputs. Owing to this complexity, studies in this domain used to be conducted more at the correlative level or focused on hormonal or genetic traits. Now, ecologically relevant, well-controlled behavioural paradigms in lab settings are being created, and new technologies for behavioural monitoring, recognition and manipulation are being implemented. These advances will enable more direct causality studies at multiple levels, including the molecular, cellular and neural circuitry levels. Most excitingly, some studies have pioneered tracking behavioural patterns, physiological parameters or even neural activities of multiple animals simultaneously. Such advancements will be particularly insightful for studying social interactions in complex and naturalistic environments.

In my view, one interesting, big-picture question in psychiatric neuroscience is: how do some drugs rapidly ameliorate or induce certain psychiatric symptoms? Studies related to this question should not be considered as standard pharmacological research on mechanism of action, but 'straight roads' towards the core brain mechanisms of psychiatric disorders. Why so? Psychiatric disorders are well known for their slow progression, complex symptoms and multiplex genetic and epigenetic factors, making it a daunting task to understand the underlying aetiologies. However, some rapid-acting drugs quickly alleviate or create a mentally disordered state, providing a convenient and clinically relevant perturbation tool to unlock the hidden doors of psychiatric diseases. For example, as antagonists of the NMDA receptor, phenecyclidine can rapidly induce symptoms of schizophrenia, whereas ketamine can rapidly alleviate symptoms of depression. Their actions imply that NMDA receptors are key players and strong target candidates for these disorders. This knowledge, combined with brain-circuit-specific information acquired through localized drug application and circuit manipulation, should shed light on the diagnosis, prevention and treatment of mental health disorders.

In terms of lines of research, I'm personally most excited about how social dominance shapes different behavioural traits, and what special pharmaceutical features enable ketamine's rapid and sustained antidepressant effects. 
Yasmin L. Hurd. Each decade has normally brought revolutionary advances to the neuroscience field and the past two decades have been exceptional. A few advances that instantly resonate with me include those that have continued to address a major challenge in neuroscience, that being understanding the heterogenous mosaic of brain cells. Recent single-cell sequencing strategies and genetic tools have revealed vast differences in the molecular profiles of cells, indicating the existence of potentially over a hundred different mammalian neuronal and non-neuronal cell types. Moreover, major technological advances combined information about the genetic phenotype of cells with optics, such as optogenetics, and enabled fine-tuned control of neuronal activity to elucidate the functional role of individual cells. From such advances, we have gained better insights into the role of distinct cells localized within discrete neural circuits to directly modulate behaviours relevant to, for example, decision-making, reward, fear and negative affect. Large steps have also been taken in acquiring knowledge about the transcriptome and epigenetic landscape of the human brain. This knowledge, taken together with data from the Human Connectome Project, which is mapping structural and functional neural connections, will significantly impact our understanding of neuropsychiatric disorders. One other notable discovery is that classic monoamine neurotransmitters, such as serotonin and dopamine, can directly attach to chromatin, moving the field to consider these neurotransmitters not only as cell-to-cell receptor mediators of intracellular signalling but also as direct regulators of gene transcription.

Several of the interesting big-picture questions relate to understanding the longterm consequences of early-life events for neuropsychiatric vulnerability. Thus, important questions relate to how we conduct comprehensive longitudinal assessments (neuroimaging, biospecimens and behavioural) of individuals - from fetal development, childhood and adolescence - and how we identify early markers of risk including epigenetic mechanisms. Some of the big-picture questions directly impact governmental policies and society, such as what are the potential adverse neural effects of cannabis and cannabinoids on mental health, and what are their potential therapeutic properties? Moreover, the recent opioid epidemic has also raised big questions of how to develop novel treatments for chronic pain conditions that are devoid of addictive properties.

In terms of lines of research, I am particularly excited about the growing use of machine-learning strategies that leverage neural-derived omics and other complex big datasets to improve prediction models about neurobiological substrates linked to specific phenotypes. The potential for enhanced precision treatment of neuropsychiatric disorders is also particularly exciting with the continued development of optogenetic, DREADSS, CRISPR and nanotechnology tools, which have the potential to be applied in humans, to target cellspecific and circuit-specific neural processes. In addition, the new research on histone monaminylation is exciting as it opens up new epigenetic processes to target therapeutically. Last, I continue to be excited about efforts to expand molecular, structural and functional knowledge about the human brain. Such knowledge is essential to improve the translation of animal studies and to improve treatment strategies. Overall, my excitement stems from research efforts that provide in-depth neurobiological knowledge about neuropsychiatric disorders that is directly relevant to the human condition and that can provide critically needed new therapeutic interventions.

Sheena A. Josselyn. Understanding how we learn and remember has fascinated philosophers and scientists for hundreds of years. In the past 20 years, there has been a seismic shift in 
how we (the field) study memory. This methodological tremor was triggered by our newfound ability to gain a firm experimental grip on the basic cellular unit of memory storage, the engram (or memory trace). The general idea that a specific memory is stored as enduring changes in the brain is centuries old, but it wasn't until the beginning of the $20^{\text {th }}$ century that Richard Semon introduced the term 'engram' to describe the neural substrate for storing and recalling memories. An experience, Semon essentially proposed, activates a small subset of cells that undergo persistent chemical and/or physical changes to become an engram. Memory retrieval occurs when sensory cues later reactive this engram. Although the idea of an engram may have been theoretically appealing, similar to hunting a mythical creature, as soon as scientists tried to experimentally grab an engram, it seemed to slip away.

Over the past 20 years, though, the development of an ever-increasing array of new tools allowed scientist not only to find, but also to manipulate, 'engram cells' across several model species. The ability to catch an engram proved to be an absolute game-changer. For instance, previously we attempted to examine the biochemical and electrophysiological substrates of memory by grinding up an entire brain region or 'listening' to the electrophysiological changes of a broad population of neurons (as in field long-term potentiation recordings) after a learning event. With engram technology, changes in engram cells alone could be studied, free from the potentially obscuring effects in non-engram cells. The mnemonic wheat could finally be separated from the chaff.

With engram technology we can now implant 'artificial memories' in mice. Indeed, a handful of labs recent studies showed it is possible to create entirely artificial percepts or memories in mice, without any external stimuli, simply by manipulating cell ensembles. These science-fiction type findings verify that we are on the right track. In addition, studying memory at the level of engrams is leading to novel insights into the very fundamental building blocks of memory. For instance, we are beginning to understand how cells (initially excitatory neurons, and now expanding to include other cell types) are allocated to an engram, how the architecture of an engram impacts memory retrieval (for instance, whether events are remembered distinctly or organized into boarder conceptual knowledge), how the passage of time or new information changes engrams, the extent to which engrams are static or dynamic, and how information becomes momentarily inaccessible (as in the tip-of-the-tongue phenomenon) or irretrievably forgotten (more permanent amnesia).

That being said, many many questions remain. For instance, exactly how (and where) is information stored in the brain? Is it stored in cell ensembles, synapses, molecules or DNA? And, more basically, what do we even mean by 'information'? The answer to these 'hard' memory questions will likely require continued cross-talk between researchers examining memory in humans, rodents, fish, flies and even in silico.

Baljit S. Khakh. Twenty years ago, neuroscience consisted largely of a cottage industry of researchers pursuing open-ended questions for which they had amassed a great deal of specialized knowledge and skills. Since then, we have witnessed the dawn of 'industrial scale neuroscience', pursued as large organized initiatives, often in private institutes. These types of projects seek to tackle big problems in defined periods. They are having an inexorable and substantial impact in the details of the science itself and, increasingly, in how others plan and do their own science. Pundits, academic institutions and funding agencies are also pondering how they can align faculty to attack key problems at a scale that is not possible in single research groups. The promise of such approaches is that progress could be rapid and efficient, but the potential risk is that laboratory research, unless thoughtfully shepherded, 
may lose some of the creativity that has and continues to drive discovery and innovation in small, hands-on research groups. Undoubtedly, both models deliver in distinct ways.

In my own field of glial biology, the availability of new genetic, optical and instrumentation tools has allowed neuroscience to be explored in ways that were previously not possible, and this has changed the field to the extent that even cellular-molecular projects can now, for example, evaluate the behavioural implications of mechanistic findings. Another development has been the growth of big data - including genomic, gene and protein expression, electrophysiology, imaging and behavioural data - and the necessity to process and analyse them with computational methods.

In relation to neurons, glia are still an understudied cell population. I think the most interesting questions being asked in my area relate to how glia regulate neural circuits, how glia contribute to behaviour, and how glia contribute to the function of the brain as a highly evolved multicellular organ and not just as a collection of neuronal circuits. Including glia into how complex functions of the brain are performed also holds the promise of shedding new light on disease mechanisms as well as brain-body and neuroimmune interactions.

I am particularly excited about the prospect of manipulating and targeting glia as endogenous neuromodulators to produce disease-related phenotypic benefits in complex brain disorders. This is a worthwhile direction to follow: pursuing neuronal mechanisms alone over the past 20 years has not been as fruitful as was once anticipated in terms of disease-modifying treatments. Additional parallel and orthogonal approaches are now also necessary.

At a broader level, I think mechanistic studies and computational neuroscience are going to be critical to make sense of the vast amount of precisely tracked, technique-driven, but essentially descriptive behavioural data that are emerging in the field as a whole.

Jürgen A. Knoblich. Like other fields, neuroscience was profoundly influenced by the arrival of the post-genome era. The ability to compile complete inventories of receptors, channels and other elements of neuronal information processing, together with emerging methods for measuring their expression and function across cell types in a comprehensive manner, have brought neuroscience to another level. A second groundbreaking development was the establishment of optogenetics. The ability to turn neurons on or off has merged functional neuroscience with cell biology. Mapping brain circuits and connecting them to specific behaviours has become possible and will fill the gap between neuroanatomy and the functional analysis of individual neurons and their electrical properties.

In neurodevelopment, my own field of research, the past two decades saw the identification of radial glia cells and their various descendants as the progenitors for all neurons in the cortex. Importantly, some subtypes of these progenitor cells are unique to primates and this has triggered a new era in which analyzing unique features of the human brain is within reach.

I am convinced that the next decade in neuroscience will be the era of the human brain. It is increasingly clear that human brains are not just enlarged versions of animal brains. They have unique features and develop in a unique way and we are at the verge of understanding why that is. We have identified human-specific cell types and are beginning to understand their role in making our brains so powerful. Genome-editing, functional genomics and organoid models can be combined to allow genetic analysis in human brain tissue models. 
Eventually, those tools will endow us with the ability to ask fundamentally important questions for understanding ourselves: what makes us human and what genetic changes are responsible for the enormous complexity and cognitive power of the human brain? What makes us individual? Why does my son think like me and where is this encoded in the genome? And why do humans with alterations in specific genes think differently and develop symptoms we call autism or schizophrenia. Besides providing fundamental biological insights, the answers to those questions will also profoundly change the medical treatment of neurological and psychiatric disorders.

Needless to say, I am particularly excited about organoids. It may be surprising to some that I do not think that they will replace animal experiments or that they are or will ever become a comprehensive model for the human brain - but they allow us to take the last step towards asking specific questions about our own brain. Currently in their infancy, I am confident that organoids will develop into models for investigating certain neurobiological processes. Establishment of organoid models for functional brain circuits and for major long-range connections within the brain are within reach and can be combined with optogenetics to probe human-specific brain features at the circuit level. I am also excited about the power of combining in vitro models with ever-improving $\mathrm{fMRI}$ brain imaging methods to understand human brain circuits.

Panayiota Poirazi. The turn of the $21^{\text {st }}$ century was marked by remarkable discoveries regarding the cellular and subcellular properties of neurons, made primarily via in vitro preparations. The rich repertoire of dendritic conductances, the compartmentalization of electrical, chemical and molecular signals by dendritic branches and their ability to support local spiking was unveiled. Empowered by computational modelling, these discoveries suggested that dendrites should no longer be viewed as passive cables but as powerful computational units that greatly expand the processing and storage capacity of individual neurons.

Major technological advances of the past two decades allow causal probing of multiscale processes that operate in the brains of behaving animals and can test these propositions. New tools allow the tracking of synaptic activity and plasticity in vivo (for example, SynTagMA and GluSnFR), the characterization of inputs to individual neurons (for example, via rabies viruses) and the selective manipulation of activity (optogenetics), often restricted to specific compartments located far from the surface (for example, via prisms), while recording from hundreds of neurons across different brain areas. Voltage indicators are also coming of age and will soon replace slower molecules, and computational modelling, driven by this slew of multimodal data, has advanced to account for dendritic processing at the single neuron and circuit levels. These new tools enable unprecedented access to the microscopic, mesoscopic and macroscopic levels of computation.

The past decade has also witnessed a closer and more fruitful collaboration between theorists and experimentalists. Numerous dendritic contributions, many of which were originally predicted by models, have been now confirmed in awake behaving animals for critical brain functions such as learning and memory, sensation, navigation, and even perception and anaesthesia.

Yet, most of our current understanding is limited to dendritic effects on single neuron processing or circuit activity. Establishing causal links between dendritic function and behaviour is the ultimate challenge. A synergistic, online approach will be critical for achieving this goal. For example, monitoring of synaptic, dendritic and neuronal activity 
from different cell types and brain areas during various behaviours could be used to constrain large-scale network models in a continuous, online manner. The models will predict how changes in dendritic properties may alter aspects of behaviour and online tests will refute or verify predictions, thus closing the loop and updating both models and hypotheses.

Although the dendrites of principal neurons are intensively studied, our understanding of these structures in different cell types and species remains poor. Exciting new discoveries suggest that interneuronal dendrites can also integrate incoming signals in non-linear ways and that human dendrites can implement advanced computations previously attributed to networks.

Adopting dendritic properties to advance artificial intelligence (Al) is another exciting line of research. There are several efforts to incorporate dendritic processing in both software (for example, Deep Neural Networks) and hardware (for example, CMOS devices) technologies with the aim of achieving brain-like performance in complex tasks such as reasoning and one-shot learning. These efforts are expected to open a whole new era in bioinspired machine learning and $\mathrm{Al}$ in the years to come.

Russell A. Poldrack. In my view, one notable development in cognitive neuroscience over the past two decades has been the advent of computational theories that are being used to both drive experimentation and interpret neuroimaging data. This has occurred across many subfields, most notably in visual object recognition, but also in content areas as diverse as decision-making, memory and motor control. Together with new methods for modelling and the analysis of neuroimaging data, these models have provided a tool to more clearly link functional imaging signals to underlying computational mechanisms.

Another important development has been a transition from the highly localizationist approaches of early neuroimaging research to a focus on the hierarchical structure and dynamics of brain networks. Much of this focus has arisen from task-free fMRI studies, which have demonstrated structured correlations with clear functional relevance at multiple levels of spatial organization. In addition, we have seen the advent of 'network neuroscience', leveraging the tools of network science to mathematically characterize brain networks. It might once have been legitimate to refer to neuroimaging as 'blobology', but in most areas of neuroimaging research those days are largely gone.

For me, one of the most interesting questions is how we can reconcile the views of brain function that are currently inherent in the computational neuroscience and network neuroscience approaches. Network neuroscience has provided important insights into the structure and dynamics of brain networks, but the methods used for these analyses generally treat 'nodes' as fungible beyond their specific location within the network. By contrast, computational neuroscience has provided insights into the specific computational functions that are performed by individual brain circuits, but has had relatively little to say about the complex dynamics that occur when many such circuits are interconnected at the scale of an entire brain. Bridging these two approaches will require a rapprochement between very different analytical approaches coming from physics and machine learning, but it will be essential to developing a fuller understanding of how the brain gives rise to high-level cognitive functions.

I am particularly excited about recent work that has begun to define the functional architecture of individual human brains with much higher precision than before. After my initial work in the MyConnectome study, a number of labs have begun using a 'deep 
phenotyping' approach in which individuals are scanned repeatedly and characterized individually. This approach has already provided new insights into the fine-grained organization of the entire human brain, highlighting both the universality and diversity of functional organization at different levels, as well as identifying new functional features that were not visible in earlier group-averaged fMRI studies. Going forward, I am particularly excited to see these approaches taken into the study of mental illness. Given the great deal of variability in the time course of mental illness, understanding the dynamics of brain function in relation to the dynamics of disease is crucial to enable mechanistic insights that can lead to new treatments.

Marco Prinz. In the past two decades, we have been eyewitnesses to a renaissance of neuroimmunology research within neuroscience. In particular, a special focus has been made on understanding the function and heterogeneity of CNS myeloid cells including parenchymal macrophages (that is, microglia) and macrophages at CNS interfaces, such as perivascular, meningeal and choroid plexus macrophages. Conversely, when I started my research on microglia in the late 1990s at the Max-Delbrück-Center (MDC) for Molecular Medicine in Berlin, neuroscience was almost exclusively neuron-centred and glial researchers were considered as rather exotic foreigners neither belonging to neuroscientists nor to immunologists.

The big bang of microglia research was certainly the development of the CX3CR1GFP mouse line by Steffen Jung in 2000 when he was a postdoc in Dan R. Littman's lab in New York. This pioneering work allowed for the first time the visualization of microglia in vivo and paved the way for several important microglia discoveries in the following years. For example, the unexpected finding that 'resting' microglia are continuously active in the living mouse brain shed new light on microglial functions in the normal brain and made clear that these cells are highly active guardians of brain homeostasis. Along this line, microglial cells were subsequently identified as key CNS intrinsic architects that shape neuronal circuits during development and homeostasis by a process called synaptic pruning that was later similarly found to be present during pathological processes, such as Alzheimer disease.

The most burning questions in neuroimmunology dealing with the innate immune arm are how microglia contribute to CNS diseases and how this contribution can be modulated. Big international genome-wide association studies in recent years have identified an ever-growing number of genetic risk factors associated with several disorders - such as multiple sclerosis, Alzheimer disease, Parkinson disease and others - that are expressed by CNS myeloid cells, like microglia. The major challenge now is to understand how intergenic variations of several loci jointly lead to changes in transcription or translation and, ultimately, contribute to disease. Hopefully, rare monogenetic disorders primarily and solely affecting local tissue macrophages (for example, disorders caused by CSF1R mutations) will help to decipher the underlying mechanisms of normal and disturbed immune regulation in the CNS and might finally facilitate the future development myeloidfocused therapeutic approaches.

There are several exciting novel technological developments that currently electrify neuroimmunologists around the globe. A new era of microglial research has just begun. The urgent need to understand microglial biology led, for example, to the development of microglia-like cells from embryonic stem cells or induced pluripotent stem cells. Further thrilling techniques in the field include brain organoids, assembloids and xenotransplantations that will hopefully permit the study of environmental and genetic 
factors in microglia-mediated CNS diseases. In the future, multi-omics approaches to profile genetic, epigenetic, proteomic and metabolomic cellular states at the single-cell level, novel animal models, imaging and fate mapping tools, patient-derived microglial cells and other innovative techniques will help us to decipher more microglial secrets around CNS development, homeostasis and disease.

Pieter R. Roelfsema. Advances in technology have driven progress in systems neuroscience in important ways over the past 20 years. New methods became available to measure and control the activity of many neurons with high precision. Another noteworthy development is the increasing influence of Al, allowing neuroscientists to better understand synaptic plasticity rules and information processing in the brain.

A major change in visual neuroscience has been the focus on the mouse as a model to study vision. Many researchers would not have predicted this in 2000 , because mice do not have a particularly well-developed visual system. The disadvantage of studying a species with poor visual acuity is offset by the many powerful tools now available for mice, including optogenetics, two-photon imaging, tracing techniques and interventions using viruses and transgenic mouse lines. Even good-old electrophysiology has been improving. Researchers used to make recordings from one cell at a time, but new probes permit the study of many hundreds of neurons simultaneously. Hence, recent papers report the activity of tens of thousands of neurons, distributed across many cortical and subcortical regions during a single behavioural task. For the development of the field, it will be important to make these novel techniques available to use in species, including non-human primates, with brains that more closely resemble human brains.

An important trend has been the move towards open science and open data, providing researchers access to large data sets. This leads to a more efficient use of resources and will increase the reproducibility of results by making it easier to evaluate the validity of the conclusions of an article. By itself, access to large amounts of data is not enough to understand the brain, however. Fortunately, neuroscientific insight has started to benefit from the progress in Al. In vision, artificial neural networks are able to transform raw sensory input into object categories; that is, they extract meaning. Importantly, the tuning of units of these artificial neural networks resembles the tuning of neurons in the brain, helping neuroscientists to understand how the many areas of the brain's own 'deep networks' contribute to the step-wise transformation of pixels into semantic codes. Furthermore, $\mathrm{Al}$ is now also inspiring insights into how connections in deep brain networks can be trained efficiently.

The enhanced understanding and accessibility of neural codes through Al also opens up new possibilities for interfacing with the brain. A recent study used Al to decode language from brain activity of humans. This methodology can be applied in the future to help people with severe forms of paralysis in their communication with the outside world. In combination with new high channel count interfaces, researchers may soon read and write to the brain with unprecedented precision, opening new avenues for therapeutic intervention. Indeed, approaches are underway to create new prostheses for blind individuals by directly writing information captured by a camera to the visual cortex, skipping the malfunctioning eyes. As soon as these high density brain reading and writing are applied in humans, there are likely to cause a new wave of scientific insights into the neuronal processes underlying the human mind. 
Tara L. Spires-Jones. Exciting new lines of investigation have emerged over the past two decades in the field of Alzheimer disease research, including understanding how pathological proteins damage the brain, why certain neurons and brain regions are vulnerable to degeneration, bridging the gap between genetic and epidemiologic risk factors and the brain changes that cause dementia, and understanding how glia contribute to neurodegeneration.

The field of dementia research has been dramatically advanced by technology over this period. For example, although the pathological lesions that define Alzheimer disease were described in the early 1900s and the ability to study pathology with histology was well established over 30 years ago, the past 20 years has seen a boom in the ability to image these pathologies in living people over time with positron emission tomography, to study the pathological proteins in exquisite detail with cryo-electron microscopy, and to examine the toxic oligomeric forms of these proteins within individual synapses in animal models and human tissue. GWASs starting in the early 2000s highlighted new pathways involved in disease pathogenesis, which are being followed up using new technologies like single-cell transcriptromics and CRISPR gene editing.

Some of the biggest changes in the field over this time frame have not been neuroscience specific. The explosion of the ability to share data has been of real benefit to neuroscientists, and this is likely to become even more important over the next 20 years.

We have also seen major changes in the way we conduct neuroscience. There is an emerging awareness that early career researchers face many difficulties in pursuing an academic career. Although much more work is needed, groups such as the FENS-Kavli Network of Excellence are striving to support people coming up in the field and to enhance the diversity and inclusivity of neuroscience. In my view, we also collaborate more in neuroscience than we did 20 years ago with collaborations across disciplines and industry, and through large strategic initiatives like the UK Dementia Research Institute, the US Brain Initiative and the EU Human Brain Project. The impending global health crisis that dementia poses and the current pandemic also highlight the continued need for scientists to engage with the public and policymakers to help develop evidence-based policies to meet societal challenges.

For the next 20 years, I am excited about translation. Perhaps I'm a hopeless optimist in the face of decades of clinical trial failures for Alzheimer disease, but I think that we are close to life-changing treatments. It is extremely gratifying and humbling that our work increasing understanding of the fundamental brain changes that cause dementia symptoms is contributing, albeit in small part, to the development of clinical approaches and trials. I'm also excited about the advancing understanding of the fundamentals of the brain and what makes us human, our ability to think, learn, remember. I agree with the European Brain Council's 'Brain mission', which places understanding the brain and treating brain disorders at the heart of a $21^{\text {st }}$ century mission analogous to the $20^{\text {th }}$ century missions to put humans on the moon.

Mriganka Sur. Two complementary fields have witnessed notable developments over the past two decades: understanding cortical dynamics at the level of brain regions and neuronal populations, and cortical plasticity at the level of synapses and clusters of synapses.

Recording the activity of large numbers of neurons, via multiphoton imaging and high-density probes, has given us novel views of brain-wide activity, at single neuron resolution, in at least small brains. Manipulating regional and cell-specific activity with 
optogenetics has revealed specific contributions of brain regions and cell classes to information processing.

Applying these tools to mice performing behavioural tasks, we have derived a computational understanding, building on previous studies in nonhuman primates, of regional circuit contributions to decisions, short-term memory and actions. At the same time, recent findings puzzlingly suggest the widespread expression of signals, especially those related to action and reward, throughout the brain. These studies point to the importance of disentangling correlative versus causal signals, and the roles of specific yet dynamic local and long-range circuits in behaviour.

High-resolution imaging of synapses and synaptic molecules has led to a richer conceptual understanding of cortical plasticity. At excitatory synapses, plasticity is largely implemented by Hebbian learning rules, whereby coactive synapses are strengthened; yet, unregulated Hebbian strengthening leads to saturated synapses and unstable cell assemblies. Thus, synaptic renormalization is necessary to stably encode information in neurons and networks.

A key advance is the realization that neuronal plasticity is implemented by multiple cooperative mechanisms: Hebbian changes at specific synapses are complemented by locally coordinated plasticity at adjacent synapses that renormalizes synaptic weights over dendritic stretches, and by longer-term synaptic scaling which further renormalizes weights over the entire postsynaptic neuron. These ideas, derived from measurements in cortical neurons, likely apply to plasticity of most neurons in the brain during development and learned behaviours in adulthood.

Plasticity is integrally related to cortical dynamics and computations. A host of molecular and cellular processes are engaged by activity to modify cortical synapses and circuits. For example, the induction of plasticity in visual cortex requires a critical level of inhibition. Plasticity is gated by modulators of attention and arousal, such as acetylcholine and norepinephrine, potentially regulating inhibitory-disinhibitory circuits. The implementation of plasticity requires signalling molecules that mediate cooperative synaptic potentiation and depression, and molecules that regulate synaptic scaling. How might particular mechanisms relate to specific circuits and tasks?

Understanding cortical plasticity and dynamics has two crucial applications. First, it provides the basis for treating brain disorders. Indeed, we are increasingly seeing imaginative proposals for treating disorders of brain development and ageing that derive importantly from basic mechanisms of plasticity and dynamics. Second, it can help build efficient computing machines. Unlike computers with von Neumann architecture where processors and memory are separate, the confluence of plasticity and processing in the same circuit elements is a core component of brain computations. Cortical plasticity almost certainly mediates our robust perceptual schemas driven by few examples, in contrast to large example set-driven, yet brittle, machine perception that is the current state-of-the-art.

Hiroki R. Ueda. Just 20 years ago, we successfully obtained a catalogue of molecules, in the form of a working draft of the human genome. Now, we are acquiring catalogues of cells in entire brains and even entire bodies in some mammals. One of the greatest contributions to these achievements has come from the remarkable development and seamless integration of tissue-clearing methods and light-sheet microscopy. Tissue-clearing methods have become increasingly high-performance, faster, safer and easier to use. Light-sheet microscopy has become more precise, with higher resolution, faster and more isotropic. As 
a result, it is now possible to perform whole-body or brain profiling of cells and circuits in mice and other mammals. In fact, a three-dimensional, single-cell atlas of the adult mouse brain is already available. In addition to the acquired positional information of cells, tissuestaining methods and image informatics are also rapidly being developed to characterize various cells in the brain and body. In the near future, we may expect a colourful single-cell atlas of adult mouse, rat and marmoset brains and bodies. Technically speaking, the completion of even the human whole brain atlas may not be a dream.

What comes after the catalogues of cells? The catalogue of molecules was followed by the emergence of a new biology field called systems biology. Systems biology at cellular to organismal levels has been hard to realize until recently, because it first requires the identification of the cellular networks where a catalogue of cells plays a fundamental role. Now that the catalogue of cells are being built and whole-brain cell profiling technology is available, we may be able to identify these cellular networks, if the aforementioned approaches are combined with perturbational technology, such as next-generation genetics (genetics without mating) and the systemic delivery of adeno-associated virus (AAV). Both these technologies do not depend on mating and hence can perturb the molecules and cells of interest to test hypotheses in vivo within a couple of weeks in mice. Identification of cellular circuits will further facilitate the analysis, control and even design of cellular networks.

Regarding lines of research, I am particularly excited about the trans-hierarchical studies to investigate the role of particular residues of specific molecules in specified cells in organisms, which is currently only possible for limited model organisms such as nematodes and fruit flies. This is because the mysteries of biological systems often come from unique and irreplaceable molecules and/or cells that play essential roles in the systems. To fully and deeply understand the uniqueness and irreplaceability of an essential molecule, it is important to investigate its underlying atomic network. Likewise, to elucidate those of a pivotal cell, it's key to study its underling molecular network. 20 years from now, by the $40^{\text {th }}$ anniversary of Nature Review Neuroscience, I hope that such trans-hierarchical identification, analysis, control and design of atomic, molecular and cellular networks will be possible in higher organisms, which will eventually lead to a wider and deeper understanding of organismal systems in mammals, primates and even humans. 


\section{Danielle S. Bassett}

Departments of Bioengineering, Electrical and Systems Engineering, Physics and Astronomy, Neurology, and Psychiatry, University of Pennsylvania, PA, USA.

\section{Kathleen E. Cullen}

Department of Biomedical Engineering, Johns Hopkins University, Baltimore, MD, USA.

\section{Simon B. Eickhoff}

1. Institute of Systems Neuroscience, Medical Faculty, Heinrich Heine University Düsseldorf, Düsseldorf, Germany.

2. Institute of Neuroscience and Medicine, Brain \& Behaviour (INM-7), Research Centre Jülich, Jülich, Germany.

\section{Martha J. Farah}

Center for Neuroscience \& Society, University of Pennsylvania, Philadelphia, PA, USA.

\section{Yukiko Goda}

RIKEN Center for Brain Science, Wako-shi, Saitama, Japan.

\section{Patrick Haggard}

Institute of Cognitive Neuroscience, University College London, London, UK.

\section{Hailan Hu}

1. Department of Psychiatry of First Affiliated Hospital, Zhejiang University School of Brain Science and Brain Medicine, Zhejiang University School of Medicine, Hangzhou,China. 2. The MOE Frontier Research Center of Brain \& Brain-machine Integration, Zhejiang Laboratory for Systems \& Precison Medicine, Hangzhou, China

\section{Yasmin L. Hurd}

Departments of Psychiatry and Neuroscience, Icahn School of Medicine at Mount Sinai, Addiction Institute of Mount Sinai, New York, NY, USA.

\section{Sheena A. Josselyn}

1. Program in Neurosciences \& Mental Health, Hospital for Sick Children, Toronto, ON, Canada.

2. Department of Psychology, Physiology \& Institute of Medical Sciences, University of Toronto, Toronto, ON, Canada.

3. Brain, Mind \& Consciousness Program, Canadian Institute for Advanced Research (CIFAR), Toronto, ON, Canada.

\section{Baljit S. Khakh}

Department of Physiology, David Geffen School of Medicine, University of California Los Angeles, Los Angeles, CA, USA.

\section{Jürgen A. Knoblich}

Institute of Molecular Biotechnology of the Austrian Academy of Sciences (IMBA), Vienna BioCenter (VBC), Vienna, Austria. 


\section{Panayiota Poirazi}

Institute of Molecular Biology and Biotechnology (IMBB), Foundation of Research and Technology-Hellas (FORTH), Heraklion, Crete, Greece.

\section{Russell A. Poldrack}

Department of Psychology, Stanford University, Stanford, CA, USA.

\section{Marco Prinz}

1. Institute of Neuropathology, Medical Faculty, University of Freiburg, Freiburg, Germany.

2. Signalling Research Centres BIOSS and CIBSS, University of Freiburg, Freiburg, Germany.

3. Center for Basics in NeuroModulation (NeuroModulBasics), Faculty of Medicine, University of Freiburg, Freiburg, Germany.

\section{Pieter R. Roelfsema}

1. Department of Vision and Cognition, Netherlands Institute for Neuroscience, Amsterdam, Netherlands.

2. Department of Integrative Neurophysiology, VU University, Amsterdam, Netherlands.

3. Department of Psychiatry, Academic Medical Centre, Amsterdam, Netherlands.

\section{Tara L. Spires-Jones}

Centre for Discovery Brain Sciences and UK Dementia Research Institute, University of Edinburgh, Edinburgh, UK.

\section{Mriganka Sur}

Picower Institute for Learning and Memory, Department of Brain and Cognitive Sciences, Massachusetts Institute of Technology, Cambridge, MA, USA.

\section{Hiroki R. Ueda}

1. Department of Systems Pharmacology, The University of Tokyo, Tokyo, Japan.

2. Laboratory for Synthetic Biology, RIKEN BDR, Suita, Osaka, Japan.

e-mail:

dsb@seas.upenn.edu

kathleen.cullen@jhu.edu

simon.eickhoff@uni-duesseldorf.de

mfarah@psych.upenn.edu

yukiko.goda@riken.jp

p.haggard@ucl.ac.uk

huhailan@zju.edu.cn

yasmin.hurd@mssm.edu

sheena.josselyn@sickkids.ca

bkhakh@mednet.ucla.edu

juergen.knoblich@imba.oeaw.ac.at

poirazi@imbb.forth.gr

poldrack@stanford.edu 
marco.prinz@uniklinik-freiburg.de

p.roelfsema@nin.knaw.nl

tara.spires-jones@ed.ac.uk

msur@mit.edu

uedah-tky@umin.ac.jp

\section{Acknowledgements}

D.S.B. is grateful to the mentees, colleagues and mentors who constantly broaden her appreciation of the diversity of scientific inquiry and deepen her excitement to pursue the most puzzling questions. K.E.C. was supported by National Institutes of Health (NIH; grant numbers DC002390, DC018061 and UF1 NS111695). S.B.E. is supported by the DFG (EI 816/16-1; El 816/21-1) and the European Union's Horizon 2020 Research and Innovation Programme under Grant Agreement No. 945539 (HBP SGA3) and 826421 (Virtual Brain Cloud). Y.G. is supported by funds from the RIKEN CBS, The Uehara Memorial Foundation and JSPS Core-to-Core Program A Advanced Research Networks. Y.L.H. is supported by grants DA050323, DA048613, DA043247 and DA030359 from the National Institute of Drug Abuse (NIDA). S.A.J. acknowledges discussions with P. W. Frankland (SickKids) and members of the Josselyn and Frankland labs for continued inspiration. P.P. thanks the members of her lab (www.dendrites.gr) for their dedication and hard work. T.L.S.-J. is funded by the European Research Council (ERC) under the European Union's Horizon 2020 research and innovation programme (Grant agreement No. 681181), the UK Dementia Research Institute - which receives its funding from DRI Ltd, funded by the UK Medical Research Council, Alzheimer's Society and Alzheimer's Research UK - and the University of Edinburgh. M.S. thanks the members of his lab for their comments. Research in the lab of M.S. is supported by NIH grants EY028219, MH085802 and DA049005, and the Simons Foundation Autism Research Initiative. P.R.R. was supported by NWO (grant P15-42 “NESTOR”), the European Union's Horizon 2020 Framework Programme Agreement No. 650003 (HBP FPA) and the Friends Foundation of the Netherlands Institute for Neuroscience.

\section{Competing interests}

P.R.R. is a co-founder of and shareholder in a neurotechnology start-up, Phosphoenix (Netherlands). T.L.S.-J. receives collaborative funding from three industry partners (Autifony, Cognition Therapeutics and one anonymous industry donor), is on the Scientific Advisory Board of Cognition Therapeutics and is an editor at several journals including founding Editor in Chief of Brain Communications. None of these influenced this piece. D.S.B., K.E.C., S.B.E., M.J.F., Y.G., Y.L.H, S.A.J., B.S.K., J.A.K., H.H., P.P., R.A.P., M.P., M.S. and H.R.U. declare no competing interests.

\section{The contributors}

Danielle S. Bassett is the J. Peter Skirkanich Professor at the University of Pennsylvania, Departments of Bioengineering, Electrical and Systems Engineering, Physics and Astronomy, Neurology, and Psychiatry. Her expertise centres on the architecture, function, design and control of complex systems, with a particular focus on large-scale neural systems in humans.

Kathleen E. Cullen is a professor of biomedical engineering at the Johns Hopkins University School of Medicine. She holds joint appointments in neuroscience and in otolaryngology. Her expertise in systems and computational neuroscience, uses state-of-the-art methodologies 
that manipulate and monitor neural circuits to understand the neural computations that predict the consequences of natural self-motion and the implications for perception and action.

Simon B. Eickhoff is a neuroimaging researcher at the Heinrich-Heine University Düsseldorf and the Forschungszentrum Jülich. At the interface between neuroanatomy, data-science and medicine, his work combines brain mapping, modelling of inter-individual differences and brain-phenotype relationships as well as machine-learning for single subject prediction of behavioural traits and precision medicine.

Martha J. Farah is a cognitive neuroscientist at the University of Pennsylvania, where she directs the Center for Neuroscience \& Society. Her current research is focused on the relations between socioeconomic status and the brain.

Yukiko Goda is a team leader at the RIKEN Center for Brain Science. Her research addresses the cellular principles by which synaptic strengths are set and dynamically modified at individual synapses in relationship to other synapses in defined neural circuits. Recent efforts in the group also target roles for astrocytes in synapse regulation.

Hailan $\mathrm{Hu}$ is a professor at Zhejiang University School of Medicine, China. Her research interest focuses on how emotional and social behaviours are encoded and regulated in the brain, with a main focus on the neural circuitry underlying depression and social dominance.

Yasmin L. Hurd is Professor of Psychiatry, Neuroscience and Pharmacological Sciences at the Icahn School of Medicine in New York and Director of the Addiction Institute in the Mount Sinai Behavioral Health System. Her research focuses on opioid abuse and the developmental consequences of early drug exposure (particularly cannabis) using multidisciplinary and translational approaches in humans and animal models.

Sheena A. Josselyn is Senior Scientist at the Hospital for Sick Children (SickKids), a Professor at the University of Toronto in Canada and a Senior Fellow in the Canadian Institute for Advanced Research (CIFAR). She is interested in understanding how the brain uses information. Her lab uses a variety of experimental techniques to probe memory function in mice.

Baljit S. Khakh is a Professor of Physiology and Neurobiology at University of California Los Angeles. His expertise centres on glial biology and the role of astrocytes in neural circuits. The Khakh laboratory develops and uses novel genetic and optical methods to explore glial biology in vivo.

Jürgen A. Knoblich is Director of the Institute of Molecular Biotechnology of the Austrian Academy of Sciences in Vienna. He is a developmental neuroscientist studying human brain development and psychiatric disorders. Researchers in his group have developed a method for growing human brain tissue in the lab. They can recapitulate human embryonic brain development during the first trimester and analyze the developmental defects leading to neurological disorders. 
Panayiota Poirazi is a neuroscientist at the Foundation for Research and Technology-Hellas in Heraklion, Crete, Greece. She uses mathematical and computational techniques to develop experimentally constrained models of single neurons and neuronal networks, aiming at inferring the contributions of dendritic computations to complex brain functions.

Russell A. Poldrack is the Albert Ray Lang Professor in the Department of Psychology and Professor (by courtesy) of Computer Science at Stanford University, and Director of the Stanford Center for Reproducible Neuroscience. His research uses neuroimaging to understand the brain systems underlying decision-making and executive function. His lab also develops neuroinformatics tools to help improve the reproducibility and transparency of neuroscience.

Marco Prinz is a neuropathologist at the University of Freiburg, Germany. Currently, his research group aims to understand myeloid cell biology in the CNS during health and disease and studies the impact of the CNS endogenous immune system on the pathogenesis of neurological disorders such as neurodegenerative and neuroinflammatory diseases.

Pieter R. Roelfsema has been Director of the Netherlands Institute for Neuroscience, Amsterdam, since 2007. He studies visual perception and perceptual organization, as well as plasticity rules. He aims to develop a neuroprosthesis for the visual cortex to restore a rudimentary form of vision in blind individuals.

Tara Spires-Jones is Professor of Neurodegeneration and a UK Dementia Research Institute Group Leader at the University of Edinburgh. Her group studies the role of synapses in brain degeneration and resilience. Using pioneering imaging techniques, her group has discovered links between genetic risk factors for Alzheimer disease and synaptic degeneration.

Mriganka Sur is a neuroscientist at the Massachusetts Institute of Technology, where he directs the Simons Center for the Social Brain. His lab studies mechanisms of brain wiring and processing, especially in the cerebral cortex, and how they go awry in disorders of brain development.

Hiroki R. Ueda is a professor at the University of Tokyo and a team leader in RIKEN BDR. He has an expertise in systems biology and focuses on mammalian sleep-wake cycles. He invented the whole-brain and whole-body clearing and imaging methods called CUBIC and the next-generation mammalian genetics for one-step production and analysis of knock out and knock in mice without crossing. 